\title{
Association of HLA and Mutated CCR5 With the Clinical Course of the Disease in Subjects With mild / moderate disease following COVID-19 infection
}

\section{Johannes C Fischer}

Heinrich Heine University Düsseldorf: Heinrich-Heine-Universitat Dusseldorf

Albrecht G Schmidt

Heinrich Heine University Düsseldorf: Heinrich-Heine-Universitat Dusseldorf

Edwin Boelke ( $\sim$ boelke@med.uni-duesseldorf.de)

Heinrich-Heine-Universitat Dusseldorf https://orcid.org/0000-0001-9112-1024

\section{Markus Uhrberg}

Heinrich Heine University Düsseldorf: Heinrich-Heine-Universitat Dusseldorf

Verena Keitel

Heinrich Heine University Düsseldorf: Heinrich-Heine-Universitat Dusseldorf

\section{Torsten Feldt}

Heinrich Heine University Düsseldorf: Heinrich-Heine-Universitat Dusseldorf Björn Jensen

Heinrich Heine University Düsseldorf: Heinrich-Heine-Universitat Dusseldorf

\section{Dieter Häussinger}

Heinrich Heine University Düsseldorf: Heinrich-Heine-Universitat Dusseldorf

Ortwin Adams

Heinrich Heine University Düsseldorf: Heinrich-Heine-Universitat Dusseldorf

E. Marion Schneider

University of Ulm: Universitat Ulm

\section{Vera Balz}

Heinrich Heine University Düsseldorf: Heinrich-Heine-Universitat Dusseldorf Jürgen Enczmann

Heinrich Heine University Düsseldorf: Heinrich-Heine-Universitat Dusseldorf Jutta Rox

Heinrich Heine University Düsseldorf: Heinrich-Heine-Universitat Dusseldorf Derik Hermsen

Heinrich Heine University Düsseldorf: Heinrich-Heine-Universitat Dusseldorf

\section{Karin Schulze-Bosse}

Heinrich Heine University Düsseldorf: Heinrich-Heine-Universitat Dusseldorf

Detlef Kindgen-Milles 
Heinrich Heine University Düsseldorf: Heinrich-Heine-Universitat Dusseldorf

\section{Wolfram Trudo Knoefel}

Heinrich Heine University Düsseldorf: Heinrich-Heine-Universitat Dusseldorf

\section{Martijn van Griensven}

Maastricht UMC+: Maastricht Universitair Medisch Centrum+

\section{Jan Haussmann}

Heinrich Heine University Düsseldorf: Heinrich-Heine-Universitat Dusseldorf

\section{Balint Tamaskovics}

Heinrich Heine University Düsseldorf: Heinrich-Heine-Universitat Dusseldorf

\section{Christian Plettenberg}

Heinrich Heine University Düsseldorf: Heinrich-Heine-Universitat Dusseldorf

\section{Kathrin Scheckenbach}

Heinrich Heine University Düsseldorf: Heinrich-Heine-Universitat Dusseldorf

\section{Stefanie Corradini}

LMU München: Ludwig-Maximilians-Universitat Munchen

\section{Alessia Pedoto}

Memorial Sloan Kettering Cancer Center

\section{Kitti Maas}

Heinrich Heine University Düsseldorf: Heinrich-Heine-Universitat Dusseldorf

\section{Livia Schmidt}

Heinrich Heine University Düsseldorf: Heinrich-Heine-Universitat Dusseldorf

\section{Olaf Grebe}

HELIOS Hospital Wuppertal: HELIOS Universitatsklinikum Wuppertal

\section{Irene Esposito}

Heinrich Heine University Düsseldorf: Heinrich-Heine-Universitat Dusseldorf

\section{Anja Erhardt}

Witten/Herdecke University: Universitat Witten/Herdecke

\section{Matthias Peiper}

Heinrich Heine University Düsseldorf: Heinrich-Heine-Universitat Dusseldorf

\section{Bettina Alexandra Buhren}

Heinrich Heine University Düsseldorf: Heinrich-Heine-Universitat Dusseldorf

\section{Christian Calles}

Heinrich Heine University Düsseldorf: Heinrich-Heine-Universitat Dusseldorf

\section{Andreas Stöhr}

Heinrich Heine University Düsseldorf: Heinrich-Heine-Universitat Dusseldorf

\section{Artur Lichtenberg}

Heinrich Heine University Düsseldorf: Heinrich-Heine-Universitat Dusseldorf

\section{Noemi Freise}

Heinrich Heine University Düsseldorf: Heinrich-Heine-Universitat Dusseldorf 


\section{Matthias Lutterbeck}

Heinrich Heine University Düsseldorf: Heinrich-Heine-Universitat Dusseldorf

\section{Amir Rezazadeh}

Heinrich Heine University Düsseldorf: Heinrich-Heine-Universitat Dusseldorf

\section{Wilfried Budach}

Heinrich Heine University Düsseldorf: Heinrich-Heine-Universitat Dusseldorf

\section{Christiane Matuschek}

Heinrich Heine University Düsseldorf: Heinrich-Heine-Universitat Dusseldorf

\section{Research}

Keywords: SARS-CoV-2, neutralizing antibodies, persistence, HLA haplotypes, chemokine receptor, CCR5, ABO blood groups, COVID-19, clinical course of the disease

Posted Date: June 16th, 2021

DOI: https://doi.org/10.21203/rs.3.rs-600023/v1

License: (a) (i) This work is licensed under a Creative Commons Attribution 4.0 International License. Read Full License 


\section{Abstract}

COVID-19, the pandemic infection caused by SARS-CoV-2, may take highly variable clinical courses, ranging from symptom-free and pauci-symptomatic to fatal disease. The goal of the current study was to assess the association of COVID-19 clinical courses controlled by patients' adaptive immune responses without progression to severe disease with patients' Human Leukocyte Antigen (HLA) genetics, the presence or absence of near-loss-of-function delta 32 deletion mutant of $\mathrm{C}-\mathrm{C}$ chemokine receptor type 5 (CCR5) and AB0 blood group antigens. We further analyzed the association of these immunogenetic background characteristics with patients' humoral antiviral immune response patterns, assessed longitudinally. The study enrolled 157 convalescent adult patients followed up for up to 250 days.

Univariate HLA analyses identified putatively protective HLA alleles (HLA class II DRB1*01:01 and HLA class I B*35:01, with a trend for DRB1*03:01) associated with reduced durations of disease and decreased (rather than increased) total anti-S IgG levels providing virus neutralizing capacity comparable to non-carriers. Conversely, analyses also identified HLA alleles (HLA class II DQB1*03:2 und HLA class I $B * 15: 01)$ not associated with such benefit in the patient cohort of this study. Hierarchical testing by Cox regression analyses confirmed the significance of the protective effect of the HLA alleles identified (when assessed in composite) in terms of disease duration, whereas $A B 0$ blood group antigen heterozygosity was found to be significantly associated with disease severity (rather than duration) in our cohort. A seeming association of a heterozygous CCR5 delta 32 mutation with prolonged disease duration suggested by univariate analyses was not confirmed by hierarchical multivariate testing.

In conclusion, the current study shows that the presence of certain "protective" HLA alleles is of even stronger association with reduced duration of mild and moderate COVID-19 than age or any other potential risk factor assessed. Prospective studies in larger patient populations assessing the impact of HLA genetics on the capacity of mounting protective vaccination responses may be warranted.

\section{Introduction:}

Genetic factors underlying the wide inter-individual variability of the clinical course of COVID-19 remain poorly characterized to date. Most clinical studies dissecting the association of genetic characteristics with COVID-19 either focus on the susceptibility to infection or on disease progression to serious morbidity or mortality. In the current study, we took the differentiated approach of assessing immunogenetic characteristics for their association with COVID-19 clinical courses as well as with adaptive antiviral humoral immune response patterns in patients who mastered the infection without progression to severe manifestations ( $\mathrm{WHO}^{\circ} 3$ or less).

Our analyses focused on immunogenetic background characteristics governing adaptive antiviral immune responsiveness and durable maintenance thereof in different ways:

Firstly, we were interested in elucidating the potential impact of CCR5 delta32 (CD195), a near-loss-offunction mutant of the C-C chemokine receptor type 5 on the COVID-19 clinical course. CCR 5 has been 
associated with susceptibility or resistance to a broad spectrum of viral disease ${ }^{1-3}$. A recent epidemiological analysis suggests that the CCR5 delta32 mutation is associated with increased susceptibility to SARS-CoV-2 infection and fatal COVID-19 outcome ${ }^{4}$. CCR5 is known to play decisive roles as a chemotactic receptor abundantly expressed on monocytes, macrophages and T-cells and its heterozygous mutation related deficiency implies impaired memory CD $4+T$-cell response ${ }^{5}$. Thus, in regard to the COVID-19 clinical course the roles of CCR5 (1) within the oral-pharyngeal immune system as the first line of antiviral immune defense and (2) as a critical receptor governing adaptively induced T-cell memory provide a compelling immune-mechanistic rationale for the putative association of the delta32 near-loss-of-function mutation of CCR5 with altered susceptibility to SARS-CoV-2 infection and COVID-19 morbidity ${ }^{6-9}$.

Secondly, we analyzed the HLA genotypes of our patient cohort. Recognition of viral antigens presented on HLA molecules to T-cell receptors is central to all T-cell mediated antiviral immune responses, both, cellular cytotoxic and humoral ${ }^{10}$. Thus, HLA haplotypes directly impact the clonal architectures of T-cell dependent adaptive immune responses targeting SARS-CoV-2. Therefore, they are the most important for shaping T- and B-cell clonal architectures. With this knowledge it is not surprising that distinct HLA alleles have been found to be associated with increased or decreased SARS-CoV-2 infectious risks and COVID-19 morbidity, respectively ${ }^{11-14}$.

In addition, the current study also considered the potential impact of $A B 0$ blood group antigens assessed at genetic level on COVID-19 morbidity. Blood group antigens were included in our genetic data sets because blood group $\mathrm{A}$ (genotype $\mathrm{AA}$ or $\mathrm{A} 0$ ) has been shown to be associated with severe disease (WHO $5-8$ ) in a genome wide association study ${ }^{9}$. However, the precise level of COVID-19 risk strictly attributable to $\mathrm{ABO}$ blood group antigens per se (rather than linked co-variates such as isoagglutinin levels or Rhesus system antigens) remains a subject of uncertainty following study outcomes assigning different levels of risk ${ }^{15-17}$.

\section{Patients, Materials And Methods}

The study was performed according to the declaration of Helsinki. The local ethics committee, University of Düsseldorf, Germany (registration and approval number 2020 -1116) authorized the study protocol. As stipulated by the study protocol, genomic AB0 blood groups, HLA-A*,-B*, -C*, -DRB1*, -DQB1*, and DPB1* genotypes (https://sci-hub.se/https://doi.org/10.1111/tan.13384) ${ }^{5}$, and presence vs. absence of the $\mathrm{C}$-C chemokine receptor type 5 (CCR5) delta 32 mutations were determined in patients consenting to the study procedures as well as to potential plasma donation. The study cohort consisted of 78 male (mean age of $42.9 \pm 13.3$ years, range 19.7-76.8 years) and 79 female potential plasma donors (mean age of $45.3 \pm 13.7$ years, range 19.9-79.5 years). Severity of disease was classified as 0 for patients without any symptoms (corresponding to WHO COVID-19 ordinal scale WHO ${ }^{\circ} 1$ ), as 1 in patients with only mild symptoms and no relevant restriction of activities (WHO 1 ), 2 for patients with restriction of activities (corresponding to $\mathrm{WHO}^{\circ} 2 \mathrm{a}$ ) and 3 for patients with more severe symptoms (corresponding to 
$\mathrm{WHO}^{\circ} 2 \mathrm{~b}$ and borderline $\mathrm{WHO}^{\circ} 3$ ). None of the patients were hospitalized. In patients with symptoms, activity restriction was confirmed by telephone interview.

\section{Antibody assessments}

Two lateral flow serological assays were used in this observational clinical study. The spike S1 protein domain was mainly selected as an antigen and recognized by IgA and IgG in all donors using the ELISA test of Euroimmun, Germany. The Roche Diagnostic test (Ig nucleocapsid) was used confirmatively. Additionally, the IgG virus nucleocapsid antigen (Euroimmun) was selected as an antigen to detect appropriate immune globulins in donors. All assays were conducted according to manufacturer's standard operating procedures/ protocols.

\section{C-C chemokine receptor type 5 (truncated) and CCR5 wild type (wt) assessment}

An amplicon-based approach to genotype the CCR5 wt and the CCR5 del32 was used to amplify exon 3 of the respective gene. Briefly, amplification of exons 2, 3, and 4 of HLA class I and HLA-DPB1 genes, and exons 2 and 3 of HLA-DRB1 and HLA-DQB1 (in parallel with selected exons of the blood group system genes $\mathrm{ABO}$ and $\mathrm{Rh}$ as well as CCR5) were carried out in six multiplex polymerase chain reactions (PCRs). After a purification step using paramagnetic beads, the amplificates underwent a second-round PCR that added sample-specific barcodes and Illumina-compatible adapter sequences. All PCR products were pooled, the resulting library was quantified using real-time PCR applying primers directed to the Illumina specific adapter (Illumina Inc., San Diego, CA) and were sequenced on a MiSeq device (Illumina). The analysis of the read sequences was performed by an in-house software (NGSSequence Analyser, Institute of Transplantation Diagnostics and Cell Therapeutics, University Hospital of Duesseldorf, Duesseldorf, Germany) approach taking into account quality control values and high coverage to automate data analysis. Sophisticated algorithms were developed to distinguish between sequencing artefacts such as cross-over products and closely related alleles, as well as for the identification of novel alleles.

In order to determine the complete HLA gene sequence in questionable results, we developed a second NGS work flow. After amplification of the HLA gene using primers directed to the $5^{\prime}$ - and $3^{\prime}$ untranslated regions (UTRs), the amplificate was fragmented, end-polished and ligated to Y-adapters. A second PCR supplemented the resulting fragments with sample-specific barcodes and Illumina compatible adapters. The samples in test were pooled, the obtained library was quantified using a real-time PCR approach and run on a MiSeq instrument (Illumina). In case of uncertain results, the alignment of the reads and the analysis were performed using the NGSengine software (GenDx, Utrecht, The Netherlands).

\section{Neutralizing antibodies testing}

Donor plasma or sera were heat-inactivated for $30 \mathrm{~min}$ at $56^{\circ} \mathrm{C}$. Two-fold serial dilutions (ranging from 1:5 to 1:10240) were prepared in $50 \mu$ volume of maintenance medium (Dulbecco's Modified Eagle Medium (Thermo Fisher), $100 \mathrm{U} / \mathrm{mL}$ Penicillin and $100 \mu \mathrm{g} / \mathrm{mL}$ Streptomycin (Gibco), 2\% Fetal Calf Serum 
(PAN Biotech)). All samples were tested in duplicate. Then, $50 \mu \mathrm{l}$ of the virus stock solution with the SARS-CoV-2 NRW-42 isolate was added to a final concentration of 280 TCID50/ml. Virus stock was stored at $-80^{\circ} \mathrm{C}$ and the infectious viral titer was determined every four weeks. Cell-free plates were preincubated at $37^{\circ} \mathrm{C}, 5 \% \mathrm{CO}_{2}$ for $1 \mathrm{~h}$ before $100 \mu \mathrm{l}$ of cell suspension containing $7 \times 10^{4} / \mathrm{ml}$ Vero cells (ATCC-CCL-81, obtained from LGC Standards), continuously checked for mycoplasma contamination and added to the donor samples. Plates were then incubated at $37^{\circ} \mathrm{C}, 5 \% \mathrm{CO}_{2}$ for 96 hours.

The serum neutralization titer was determined by microscopic inspection as the highest serum dilution without virus-induced cytopathic effect (CPE). As positive controls, two previously tested sera from SARSCoV-2 infected individuals were tested at the same time in each run. One high-titer control (NT 1:640) and one medium-titer control (NT 1:160) were used for validation of each assay. Inter-assay and intra-assay variation were determined with these control sera showing a maximum deviation of only one dilution step. Additionally, serum from SARS-CoV-2 uninfected individuals and cells without serum served as negative controls to confirm virus-induced CPE. Omission of serum and virus solution served as control for cell growth during the conducted protocol.

A neutralization titer of 1:160 was defined as the threshold titer for binary representations of virus neutralization capacity allowing the potential plasmapheresis product to serve as a therapeutic agent. As the protocol reports the highest serum/plasma dilution with neutralization of $100 \%$ of infectious virus particles, the reported titers are a conservative estimate of the neutralizing capacity of donor plasma. The neutralization titers of 118 donors with a confirmed positive test result for SARS-CoV-2 by PCR or a reported episode of symptoms consistent with COVID-19 and a positive result in serological testing for anti-SARS-CoV-2 were tested in the current study.

\section{Statistics}

Statistical inference between two groups of interest was assessed by t-test statistics. Comparison of duration-of-infection times between groups of interest was performed by Kaplan-Meier curves and with two-sided log rank tests. Multivariable Cox regression analysis was performed to identify significant predictors for duration-of-infection. Effects in the multivariate Cox regression models were quantified by hazard ratio estimates with corresponding $95 \%$ confidence intervals. All p-values were calculated with two-sided tests and p-values less than 0.05 were considered statistically significant. Analyses were conducted using IBM SPSS Statistics for Windows (Version 22.0. Released 2013, Armonk, NY: IBM Corp) as well as R (3.6.3, https://www.r-project.org/).

\section{Results}

This exploratory observational study enrolled a total of $n=157$ adult Caucasian COVID-19 convalescent patients, who were followed-up in an out-patient setting. Patient-age ranged from 20 to 80 years (mean age 44 years) with a balanced distribution of female $(n=79)$ versus male $(n=78)$ patients. Study participants had suffered and recovered from mild to moderate COVID-19 disease, whereby approximately $38 \%$ of patients had experienced mild (WHO 1 and $2 \mathrm{a}$ ) and $52 \%$ moderate COVID-19 
disease $\left(\mathrm{WHO}^{\circ} \mathrm{2b}\right.$ and 3 ). Patients who had experienced mild disease tended to be younger (age range 19.7-79.5 years, mean 41.2 years, median 38.3 years) than patients experiencing moderate disease (age range 20.7-66.5 years, mean 45.2 years, median 49.6 years, $p<0.028)$. No other notable differences in terms of demographic characteristics, comorbidities or concomitant medications were observed between cohorts recovered from different severity grades of COVID-19. This statement applies to all sub-group analyses reported here, unless specifically noted otherwise.

In this convalescent patient cohort, S1-specific IgA antibody levels were barely detectable (ratio $<0.8)$ or low (ratio $0.8-1.0$ ) in 41 of the 130 tested individuals yet markedly elevated (ratio $\geq 1.1$ ) in the remaining 89 patients. IgA antibody levels were lower in blood samples taken later than 32 days following the end of symptoms (Fig. 1a). These IgA antibody kinetics are principally in line with earlier longitudinal analyses of IgA responses against SARS-CoV-2 antigens ${ }^{18,19}$. No apparent correlation between IgA antibody titers and severity of disease was observed in our study population as a whole (Fig. 1a).

S1- (Fig. 1b) and N- (Fig. 1C) specific IgG antibody levels were scattered over a large concentration range over the whole study period. However, in contrast to anti-S IgA levels anti-S IgG titers were higher in individuals with more severe disease (Fig. 1b and Fig. 1d), in accordance with findings reported before ${ }^{19-}$ 21. Throughout the study observation period of up to 240 days after the end of symptoms, IgG antibody levels tended to be stable or even elevated in 29 of the 42 subjects tested, whereas they decreased in the remaining 13 subjects (Fig. 2b, c). In all patients in whom elevated antiviral IgG antibodies had been observed at earlier time-points, the respective anti-S IgG antibodies remained detectable at the last observation recorded.

Of note, 81 of 118 samples analyzed in this study (68.6\%) neutralized virus at the prospectively defined minimum threshold titer or higher. This proportion of samples exhibiting relevant virus neutralizing capacity is in line with results of a previous study in a similar cohort of convalescent COVID-19 patients ${ }^{16}$. Supplementary Fig. 5 displays the correlation between neutralizing antibody titers and anti-S IgG levels. While a certain level of correlation between the two parameters is found $\left(R^{2}=0.299\right.$.), the wide interindividual scatter allows for the conclusion that total anti-S IgG levels are not adequately predictive of virus neutralizing capacity. Thus, at the individual patient level, total ELISA determined anti-S IgG levels do not permit to reliably discern high or low levels of neutralizing anti-S IgG antibodies.

Sensitivity analyses for patients' gender and patients' age did not identify any statistically significant and clinically meaningful differences between the respective cohorts, neither in regard to antiviral antibody titers nor their kinetics (Suppl Fig. 1). For a potential exception, a consistent trend in younger patients (< 32 years of age) towards lower antiviral antibody titers compared to older patients was noted, potentially indicative of a more prominent role of adaptive T-cell mediated rather than humoral antiviral immune responses in younger patients and/or anti-S IgG antibody epitope specificities of higher protective capacity yet the trend was not highly significant. The trend nevertheless affirms that low anti-S antibody titers cannot be readily interpreted as lack of antiviral protection, specifically when observed in younger 
patients. This interpretation was supported by the distribution of antiviral sero-negative patients across age groups.

Next, we analyzed the impact of the CCR5 delta 32 mutation on COVID-19 morbidity and the humoral antiviral immune response in 126 of the 157 individuals. The median concentrations of S1-specific IgA antibodies and of $\mathrm{N}$-specific IgG antibodies were significantly $(\mathrm{p}<0.01$ and $\mathrm{p}<0.042$, respectively) lower (Fig. 3a) in the 23 heterozygous carriers of the CCR5 delta 32 mutation, as compared to the 103 tested individuals carrying wild type CCR5. However, S1-specific IgG antibody levels did not differ between carriers of the CCR5 delta 32 mutation as compared to carriers of only CCR5 wildtype alleles (Fig. 3a) while duration of disease was prolonged significantly according to univariate analysis $(p<0.04)$ in the presence of the CCR5 delta 32 mutation (Fig. 3b). Our data do not provide evidence that the heterozygous presence of the CCR5 delta 32 mutation is associated with more severe disease, i.e. we did not find this mutation over-represented in cohorts with more severe disease, with 8 of 23 carriers of the mutation (35\%) experiencing mild disease.

Previously, the HLA class II allele DRB1*03:01 has been reported to be associated with a potentially more favorable outcome of SARS-CoV-1 in two Asian populations ${ }^{12-14}$. In this study, where 119 Caucasian individuals were HLA typed, we also observed that those 20 of them expressing the DRB1*03:01 allele tended to have shorter disease durations. However, the difference did not reach statistical significance (Fig. 4a).

In addition, the HLA class II allele DRB1*01:01 (Fig. 4b) and the HLA class I allele B*35:01 (Fig. 4c), which were expressed in 19 and 15 individuals respectively, were also associated with a significantly shorter disease duration $(p<0.003$ and $p<0.014$, respectively). The disease duration was even shorter in the six individuals expressing two of the identified "protective" HLA alleles (Fig. 4d). However, 18 of the individuals expressing the DRB1*01:01 allele co-expressed the HLA-DQA1*01:01 DQB1*05:01 allele. Therefore, the association of one or both alleles with disease duration remains to be determined. It is noteworthy that the "protective" HLA alleles share the characteristic of binding a large number of the peptides putatively derived from 9621 SARS-CoV-2 viral proteins at a high cumulative calculated HLAallele affinity score by "in silico" analysis, specifically, the HLA class II alleles ${ }^{22}$.

DRB1*01:01, DRB1*03:01 and DQA1*01:01 DQB1*05:01 putatively bind 1758, 997 and 837 of the 7903 HLA class II binding peptides presumably derived from the SARS-CoV-2 viral proteins at a cumulative calculated HLA allele affinity score of at least 1422, 1035 and 918, respectively. The HLA class I allele $B \star 35: 01$ putatively binds 183 of the $3540 \mathrm{HLA}$ class I binding peptides presumably derived from the SARS-CoV-2 viral proteins at a cumulative calculated HLA allele affinity score of at least 46.8 .

In contrast, the disease durations tended to be longer $(p<0.061$ and $<0.095$, respectively) in individuals who expressed the HLA class II allele DQB1*03:02 (Suppl Fig. 3a) or the HLA class I allele B*15:01 (SuppI Fig. 3b). The latter two alleles share the characteristic of binding a low number of the peptides derived from the SARS-CoV-2 viral protein at a low cumulative calculated HLA allele affinity score. Specifically, 
the $B * 15: 01$ allele would only bind 75 of the 3540 HLA class I binding SARS-CoV- 2 peptides at a cumulatively calculated HLA allele affinity score lower than 21.8. The DQB1*03:02 allele putatively binds 574 of the 7903 HLA class II binding SARS-CoV-2 peptides at a cumulatively calculated HLA-allele affinity score lower than 619.

No significant differences in the titer of neutralizing antibodies (Fig. $5 \mathrm{~d}$, i) or in the plasma concentration of S1-specific IgA antibodies (Fig. 5a, f) were found between the groups of individuals expressing the "protective" HLA alleles and of those individuals who did not. In contrast, the plasma concentrations of the S1-specific IgG (Fig. 5b, g) and N-specific (Fig. 4c, h) antibodies were significantly lower in the group of individuals who expressed the "protective" HLA alleles $(p<0.028-<0.004,<0.035-0.004$ depending on time point after symptom onset, respectively). This finding in conjunction with the lack of difference in the titer of neutralizing antibodies suggests that individuals expressing "protective" HLA alleles produce antibodies with higher avidity.

Blood group antigens are the third category of genetic characteristics analyzed in this study. The blood group $A$ (genotype $A A$ or $A 0$ ) has been recently shown to be associated with severe disease $\left(\mathrm{WHO}^{\circ} 5-8\right)$ ${ }^{9}$. Furthermore, $A B 0$ blood group isoagglutinins have been shown to inhibit S-protein- ACE2 interactions 23. In our cohort, the agglutination rate of incompatible blood group erythrocytes was higher in the 40 homozygous 00 individuals and in the 10 homozygous $A A$ individuals than in the 54 heterozygous $A 0,13$ heterozygous $\mathrm{B} 0$ and in the $5 \mathrm{AB}$ individuals.

The 22 AB0 homozygous individuals had a significantly $(p<0.044)$ shorter disease duration than the 21 heterozygous individuals, when the infection event affected less than 3 individuals (Fig. 6a). AB0 heterozygosity was associated with a higher risk for more severe disease (OR 2.95, 95\% Cl 1.06 to 8.19, p $<0.046$ ). Interestingly, when 3 or more people were co-infected, the risk for more severe disease tended to increase to a similar extent ("super spreading event" putatively associated with higher virus load) (Fig. 7).

In a Cox regression analysis including the covariates gender, age, the "protective" HLA alleles (as defined above), the class I HLA alleles Bw4 and C2 (introduced into this analysis, in addition, as they bind to distinct killer-cell immunoglobulin-like receptors [KIRs] thought to play key roles in the first line innate antiviral immune defense by natural killer-cells ${ }^{24}$ ), heterozygous CCR5 delta 32 mutation, as well as ABO blood group allele homozygosity, "protective" HLA alleles emerged as the strongest predictors of short disease duration (HR 1.5, 95\% Cl: 1.1-2.1) (Fig. 8a, b, c). In the subgroup of 51 individuals suffering from $\mathrm{WHO}^{\circ} 1$ and $\mathrm{WHO}^{\circ} 2 \mathrm{a}$ (mild) disease, "protective" HLA alleles, absence of CCR5delta 32 mutation and male gender were predictors of shorter disease duration (Fig. 8b).

\section{Discussion:}

COVID-19 clinical courses are highly variable between patients, with the immune mechanisms underlying this variability still incompletely understood. Understanding whether and to which extent this clinical variability is associated with given patient's immunogenetic background characteristics is of utmost 
importance for a variety of reasons: Firstly, awareness of genetically associated risk may inform individual patient's prognosis and, in consequence, prophylactic and therapeutic strategies. Secondly, a deeper understanding of the association of immunogenetics and adaptive mastery of infection may allow to better assess convalescent plasma donations for their protective capacity in the future. Thirdly, with SARS-CoV-species specifically and corona viridae in general undergoing continuous evolution, it may become critical to have established the relevance of immunogenetic background characteristics in the current pandemic in order to have access to better targeted and more effective early intervention strategies in future outbreaks. Last not least, the S-protein targeted vaccination programs currently launched worldwide immediately raise the question whether and to which extent protective vaccination responses may be impacted by a patient's immunogenetic backgrounds, responding to a novel viral antigen eliciting both de novo adaptive immune responses and expanding pre-existent cross-reactive adaptive immunity.

The current study offers partial answers and may thus help to chart out potential directions of future investigations providing conclusive answers, alongside ongoing vaccination campaigns.

Firstly, this observational study in 157 adult convalescent COVID-19 patients affirms that total anti-S IgG titers in response to SARS-CoV-2 infection are highly variable interindividually and are not adequate to reliably assess the level of antiviral immune protection in a given patient. Our study found patients immediately upon recovery from infection without detectable (16\%) or very low (8\%) anti-S IgG levels. We interpret this finding to indicate a pre-dominantly cellular antiviral immune response or better protective anti-S IgG epitope specificities in these patients. Secondly, we observed a partial dissociation of anti-S IgG titers and the capacity of virus neutralization, suggesting that total anti-S IgG levels may represent mixtures of both protective and non-protective epitope specificities. The systematic dissection of epitope specificities may deliver assay methodologies exclusively assessing antivirally protective anti-S antibodies in the future.

The above data are in line with a recently reported study by Klein et $\mathrm{al}^{20}$ in a similar convalescent cohort showing that total anti-S IgG titers are not accurate for confirming samples that are negative for neutralizing antibody responses. The assessment of this earlier study did not change when total anti-S IgG titers were replaced by IgG antibody titers directed to the $S 1$ or to the receptor binding domain of the $S$ protein 20 .

Importantly, the current study sheds new light on the association of immunogenetic background characteristics with the clinical course of COVID-19 as well as with the immune response thereto. Based on biologic functions of CCR5 established already, our data allow to outline an immune-mechanistically consistent interpretation of the role of the CCR5 delta32 deletion mutant in COVID-19. Based on univariate analyses, we observed that heterozygous carriers of this near-loss-of-function mutant suffered from disease of prolonged duration rather than more severe disease. Significantly reduced anti-S IgA levels in mutant carriers suggested that the nasopharyngeal humoral adaptive immune response is reduced in these patients, in line with the established biologic functions of CCR5 as a chemotactic 
receptor, and also in line with a recent epidemiological study suggesting increased susceptibility of CCR5 delta32 mutant carriers to SARS-CoV-2 infection ${ }^{4}$. We neither observed a difference in anti-S IgG level nor in disease severity between patients carrying mutant and wildtype CCR5.

Of note, the proportion of heterozygous carriers of the CCR5 delta32 mutant within the current study population was small $(n=23)$ and homozygous carriers were not represented. This may have impacted the strength of associations observed and certainly contributed to the fact that hierarchical multivariate (Cox regression) analyses did not confirm the association observed by univariate analyses. It remains to be seen, in consequence, whether future studies also representing homozygous carriers of the CCR5 delta mutation as well as larger patient cohorts will confirm the association between CCR5 delta32 mutant and COVID-19 disease observed in the current exploratory study.

Most importantly, our data identify two HLA class II alleles (DRB1*01:01 and DRB1*03:01) and one HLA class I allele ( $\left.B^{\star} 35: 01\right)$ associated with reduced COVID-19 duration compared to patients not carrying these alleles. Notably, the association observed with "protective" HLA alleles is stronger than the association with age, i.e. the parameter generally accepted to be best predictive of COVID-19 clinical risk. Further, one of the HLA class II alleles (DRB1*03:01) has been identified as partially protective in earlier SARS-CoV-1 as well as SARS-CoV-2 studies before ${ }^{12-14,25}$. Intriguingly, "in silico" analysis assign high cumulative calculated HLA-allele affinity scores to the three protective HLA alleles identified by the current study, adding to the biological plausibility of the association observed. The identification of protective alleles belonging to both HLA class I and class II is in line with the general immune-mechanistic concept that both CD4 helper cells (interacting via HLA class II with antigen presenting cells) and CD8 cytotoxic cells (interacting via HLA class I) play important roles in SARS-CoV-2 antiviral immune responses ${ }^{26}$. Of note, we did not find any similar association of COVID-19 duration (or severity) with class I HLA alleles (C2, Bw4) binding to distinct killer-cell immunoglobulin-like receptors thought to play key roles in the firstline innate antiviral immune response. Our analyses also identified two HLA alleles (HLA class II DQB1*03:2 und HLA class I B*15:01) associated with trends towards prolonged duration of disease, whereby $B^{\star} 15: 01$ had been noted to be associated with increased susceptibility to severe disease before $^{27}$.

Of importance, our study found protective HLA alleles not associated with increased yet rather with decreased total anti-S IgG antibody levels, once again illustrating the lack of utility of this measure as a surrogate of antiviral immune protection.

The dissociation of total anti-S IgG levels and disease controlling immune response, as observed in the current study, may be of far-reaching implication for the future evaluation and therapeutic practice of convalescent plasma therapy.

Our data strongly suggest, in line with a recent study ${ }^{20}$, that exclusive reliance on total anti-S IgG titers does not adequately account for the inter-individual variability of protective versus less protective anti-S antibody idiotype titers in a given sample. The heterogeneity of thus-defined convalescent plasma 
samples in terms of their immune-protective capacity may have contributed to variable levels of benefit generally observed from convalescent plasma therapy, in the past ${ }^{28}$. The analyses of blood group antigens in the current study are in line with earlier observations that $A B 0$ heterozygosity - which denotes to $90 \%$ of individuals with the phenotype $A$ - is associated with more severe clinical courses of COVID- 19 . In fact, $A B 0$ heterozygosity emerged as the only genetic parameter significantly associated with more severe (rather than prolonged) disease, in our analyses. However, case numbers in our study are too small to rule out other genetic associations given the interindividual heterogeneity of clinical course and large spectrum of possible confounders.

In summary, the current study identifies immunogenetic background characteristics, specifically distinct HLA genotypes, as being strongly associated with naïve host's mastery of infection with the SARS-CoV-2 neo-virus, with this association even stronger than the association with patients' age.

This study is associated with a number of limitations. This is an observational, non-interventional, uncontrolled study of exploratory design adequate for hypothesis generation yet neither designed nor powered for confirming prospectively defined hypotheses. The population deliberately consisted of patients with mild to moderate rather than severe disease in order to focus analyses on effective antiviral immune response patterns not impacted by fundamentally aberrant virus-induced immune phenomena which characterize COVID-associated acute respiratory syndrome and life-threatening disease. This dissection was introduced because it is reasonable to assume that serious COVID-19 is dominated by virally determined molecular and cellular immune mechanisms which are distinct from those relevant to preemptive mastery of infection, as is the focus of this investigation. A limitation remains, nevertheless, that patients with WHO grade $\geq 4$ are not represented in this study. Even though data collection and analysis followed a prospectively defined statistical and analysis plan, blood sampling reflected routine practice and did not follow a pre-specified sampling schedule. To control for the testing of multiple variables, key conclusions were subjected to hierarchical Cox regression analysis. Further investigations in larger, prospectively defined populations are needed.

\section{Abbreviations}

CCR5: C-C chemokine receptor type 5

CD 4: cluster of differentiation 4

COVID-19: Coronavirus disease 2019

CP: convalescent plasma

e.g.: exempli gratia

ELISA: Enzyme-linked Immunosorbent Assay

Ig: immunoglobulin 
IL: interleukin

IQR: inter quartile range

MCP: monocyte chemo-attractant protein

PCR: polymerase chain reaction

SARS-CoV-2: severe acute respiratory syndrome coronavirus 2

WHO: world health organization

\section{Declarations}

\section{Authors' contribution}

Author contribution: JCF, EB, CM, AS developed the idea of this investigation, JCF, VB, JE, JT, OA, MS, KZ, DH, KSB, TF, BJ, DH, VK, DKM, WTK, MvG, WB, JH, BT, CP, LS, AGS, KS, SC, AP, KM, LS, OG, EB, AE, IE, MP, $\mathrm{CM}, \mathrm{AS}, \mathrm{CC}, \mathrm{DH}, \mathrm{AR}, \mathrm{NF}, \mathrm{ML}$ wrote parts of the manuscript. JCF, CM and $\mathrm{KZ}$ did the literature research and prepared the data for analysis. JCF prepared the figures. KZ, EB, JCF, CM and MS contributed significantly to the discussion and the interpretation of the results. DH, KSB, OA, JT performed the laboratory tests. All authors read and approved the final manuscript.

\section{Competing interests}

The authors declare that they have no competing interests.

\section{Consent for publication}

All authors gave consent for the publication.

\section{Availability of data and materials}

All data and materials can be accessed via CM and FM.

\section{Ethics approval and consent to participate}

The study was approved by the local ethics board registration and approval number 2020-1116.

\section{Funding}

(Un-)Restricted Grant by "Kreative gegen Krebs STAR eV" (https://kreativegegenkrebs.org/; https://www.lifelolli.com/)

\section{References}


1. Ellwanger JH, Kulmann-Leal B, Kaminski VL, Rodrigues AG, Bragatte MAS, Chies JAB. Beyond HIV infection.

2. Neglected. and varied impacts of CCR5 and CCR $5 \Delta 32$ on viral diseases. Virus Res. 2020;286:198040.

3. Kindberg E, Mickiene A, Ax C, et al. A deletion in the chemokine receptor 5 (CCR5) gene is associated with tickborne encephalitis. J Infect Dis 2008;197.

4. -9 .

5. Lim JK, Louie CY, Glaser C, et al. Genetic deficiency of chemokine receptor CCR5 is a strong risk factor for symptomatic West Nile virus infection.

6. a meta-analysis. of 4 cohorts in the US epidemic. J Infect Dis. 2008;197:262-5.

7. Panda AK, Padhi A, Prusty BAK. CCR5 Delta32 minorallele is associated with susceptibility to SARSCoV-2 infection and death.

8. An epidemiological investigation. Clin Chim Acta. 2020;510:60-1.

9. Martín-Leal A, Blanco R, Casas J, et al. CCR5 deficiency impairs CD4(+) T-cell memory responses and antigenic sensitivity through increased ceramide synthesis. The EMBO journal 2020;39.

10. e104749..

11. Rijkers G, Vervenne T, van der Pol P. More bricks in the wall against SARS-CoV-2 infection.

12. involvement of gamma. 9delta2 T cells. Cell Mol Immunol. 2020;17:771-2.

13. Zheng M, Gao Y, Wang G, et al. Functional exhaustion of antiviral lymphocytes in COVID-19 patients. Cell Mol Immunol 2020;17.

14. -5 .

15. Pauza CD, Poonia B, Li H, Cairo C, Chaudhry S. gammadelta T Cells in HIV Disease.

16. Past $P$, Future. Frontiers in immunology. 2014;5:687.

17. Ellinghaus D, Degenhardt F, Bujanda L, et al. Genomewide Association Study of Severe Covid-19 with Respiratory Failure. N Engl J Med 2020.

18. Nelde A, Bilich T, Heitmann JS, et al. SARS-CoV-2-derived peptides define heterologous and COVID19-induced T cell recognition. Nat Immunol. 2021;22:74-85.

19. Pisanti S, Deelen J, Gallina AM, et al. Correlation of the two most frequent HLA haplotypes in the Italian population to the differential regional incidence of Covid-19. J Transl Med. 2020;18:352.

20. Toyoshima Y, Nemoto K, Matsumoto S, Nakamura Y, Kiyotani K. SARS-CoV-2 genomic variations associated with mortality rate of COVID-19. J Hum Genet. 2020;65:1075-82.

21. Ng MH, Lau KM, Li L, et al. Association of human-leukocyte-antigen class I ( $\mathrm{B}^{\star} 0703$ ) and class II (DRB1*0301) genotypes with susceptibility and resistance to the development of severe acute respiratory syndrome. J Infect Dis. 2004;190:515-8.

22. Chen Y-MA, Liang S-Y, Shih Y-P, et al. Epidemiological and Genetic Correlates of Severe Acute Respiratory Syndrome Coronavirus Infection in the Hospital with the Highest Nosocomial Infection 
Rate in Taiwan in 2003. J Clin Microbiol. 2006;44:359-65.

23. Golinelli D. On the association between the ABO blood group and COVID-19 susceptibility. Blood Transfus 2020.

24. Zietz M, Zucker J, Tatonetti NP. Associations between blood type and COVID-19 infection, intubation, and death. Nat Commun. 2020;11:5761.

25. Hoiland RL, Fergusson NA, Mitra AR, et al. The association of ABO blood group with indices of disease severity and multiorgan dysfunction in COVID-19. Blood Adv. 2020;4:4981-9.

26. Mariën J, Ceulemans A, Michiels J, et al. Evaluating SARS-CoV-2 spike and nucleocapsid proteins as targets for antibody detection in severe and mild COVID-19 cases using a Luminex bead-based assay. J Virol Methods. 2020;288:114025.

27. Seow J, Graham C, Merrick B, et al. Longitudinal observation and decline of neutralizing antibody responses in the three months following SARS-CoV-2 infection in humans. Nat Microbiol. 2020;5:1598-607.

28. Klein SL, Pekosz A, Park HS, et al. Sex, age, and hospitalization drive antibody responses in a COVID19 convalescent plasma donor population. J Clin Invest. 2020;130:6141-50.

29. Iyer AS, Jones FK, Nodoushani A, et al. Persistence and decay of human antibody responses to the receptor binding domain of SARS-CoV-2 spike protein in COVID-19 patients. Science immunology 2020;5.

30. Yarmarkovich M, Warrington JM, Farrel A, Maris JM. Identification of SARS-CoV-2 Vaccine Epitopes Predicted to Induce Long-Term Population-Scale Immunity. Cell Rep Med. 2020;1:100036.

31. Guillon P, Clément M, Sébille V, et al. Inhibition of the interaction between the SARS-CoV spike protein and its cellular receptor by anti-histo-blood group antibodies. Glycobiology. 2008;18:1085-93.

32. Maucourant C, Filipovic I, Ponzetta A, et al. Natural killer cell immunotypes related to COVID-19 disease severity. Science immunology 2020;5.

33. Littera R, Campagna M, Deidda S, et al. Human Leukocyte Antigen Complex and Other Immunogenetic and Clinical Factors Influence Susceptibility or Protection to SARS-CoV-2 Infection and Severity of the Disease Course. The Sardinian Experience Frontiers in immunology. 2020;11:605688.

34. Balz V, Fischer J, Krause S, Enczmann J. A novel HLA-B variant, HLA-B*14:02:16, discovered by amplicon-based next-generation sequencing. Hla. 2018;92:323-4.

35. Novelli A, Andreani M, Biancolella M, et al. HLA allele frequencies and susceptibility to COVID-19 in a group of 99 Italian patients. Hla. 2020;96:610-4.

36. Casadevall A, Grossman BJ, Henderson JP, et al. The Assessment of Convalescent Plasma Efficacy against COVID-19. Med (N Y). 2020;1:66-77.

\section{Figures}



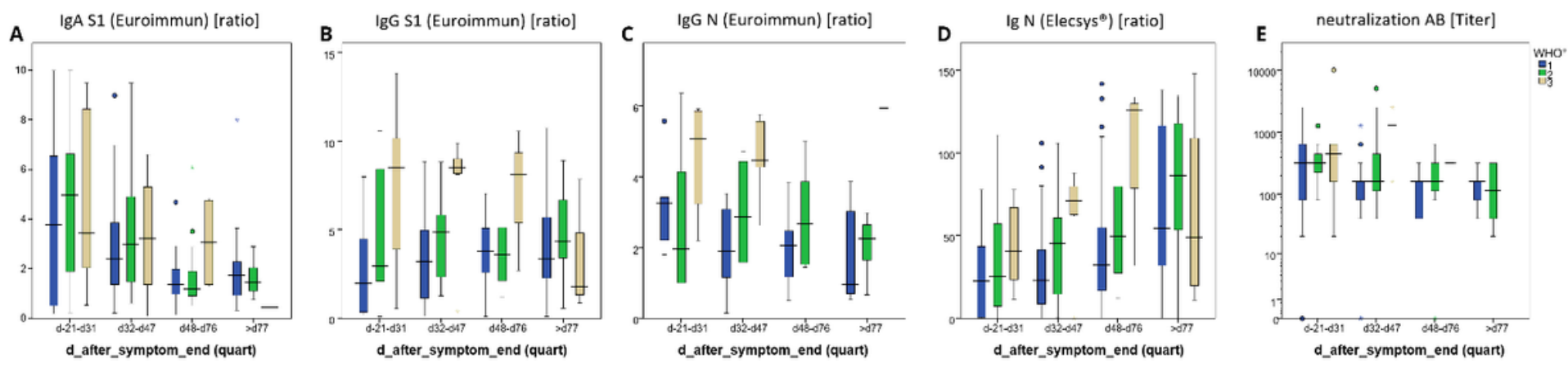

\section{Figure 1}

SARS-CoV-2 viral protein-specific antibody levels in plasma drawn up to 120 days following the end of the symptoms from individuals with different disease severity. Disease severity was according to WHO classification (Blue indicates $\mathrm{WHO}^{\circ} 1$, green $\mathrm{WHO}^{\circ} 2$, gold $\mathrm{WHO}{ }^{\circ} 3$ ). Shown are the results of the first blood draw after including the individual into the study. Panel A shows $\mathrm{S} 1$ protein-specific IgA antibody levels, measured with the Euroimmun assay. Results are expressed as ratio. Panel B shows S1 proteinspecific IgG antibody levels, measured with the Euroimmun assay. Results are expressed as ratio. Panel $\mathrm{C}$ shows nucleocapsid (N)-specific IgG antibody levels measured with the Euroimmun assay. Results are expressed as ratio. Panel D shows $\mathrm{N}$ - specific lg antibody levels detected with Elecsys ${ }^{\circledR}$, Roche. Panel $\mathrm{E}$ shows the SARS-CoV-2 serum neutralization titer, determined by microscopic inspection as the highest serum dilution without virus-induced cytopathic effect. Outliers are presented as circles (more than 1.5 IQR out of the box) or stars (more than 3 IQR out of the box). Day after end of symptoms was divided into quartiles (d-20 to d31, d32-d47, d48-d76, > d77, range between d-20 to d120).
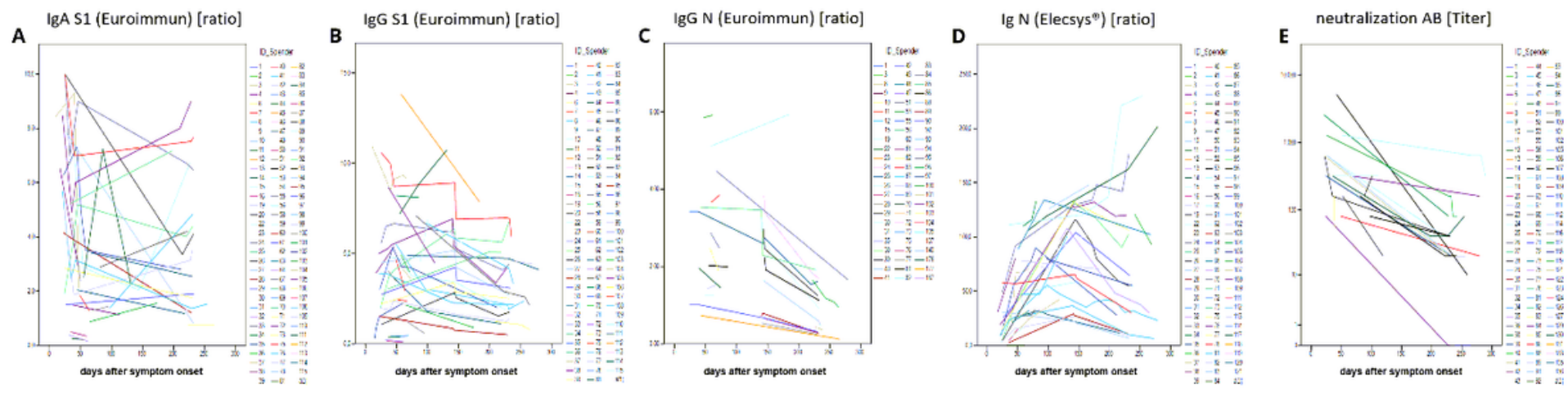

\section{Figure 2}

SARS-CoV-2 viral protein-specific antibody levels in plasmas sequentially drawn from infected individuals between day 10 and day 188 following symptom onset. SARS-CoV-2 viral protein-specific antibody levels were determined as described in the legend of Figure 1. 

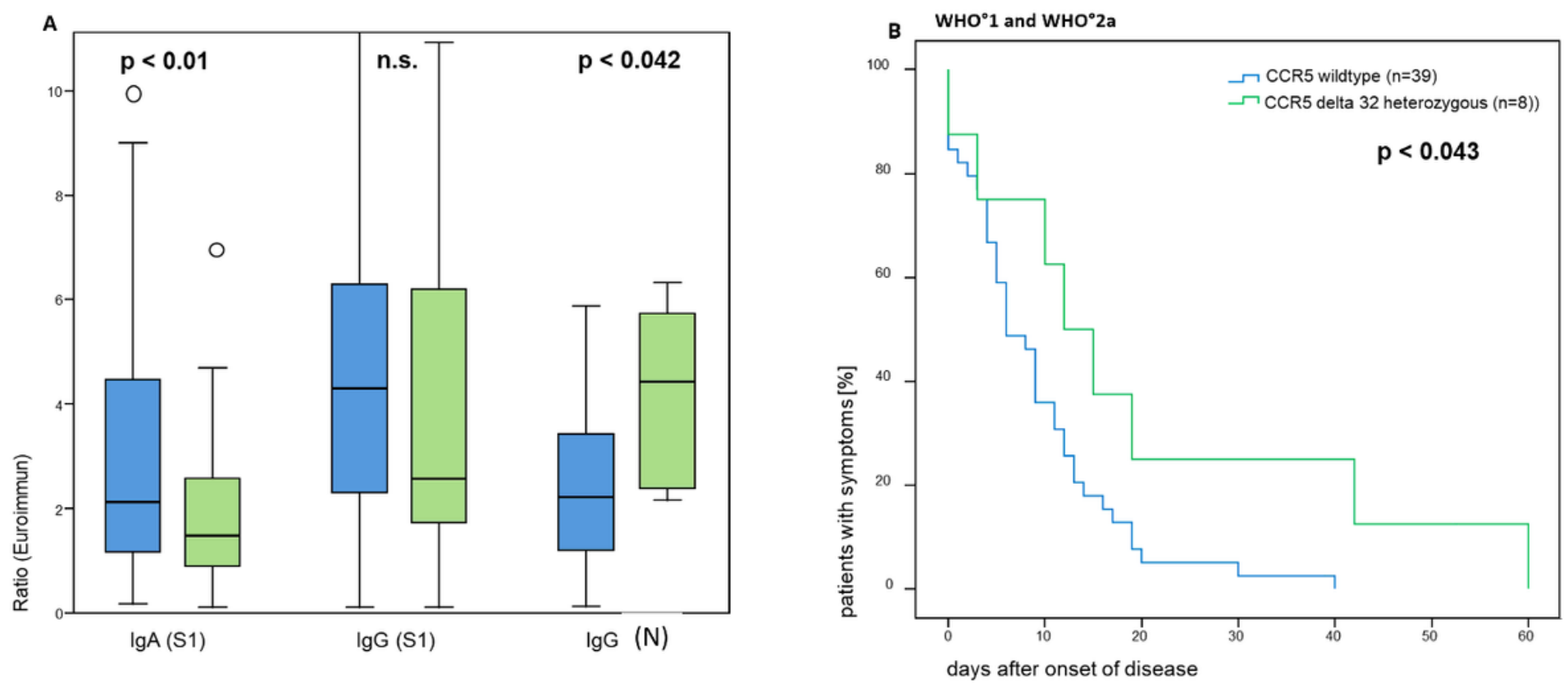

Figure 3

Significantly lower SARS-CoV-2 viral protein-specific antibody levels and significantly longer disease duration in individuals carrying the CCR5 delta 32 mutation heterozygously with $\mathrm{WHO}^{\circ} 1$ and $\mathrm{WHO}^{\circ} 2 \mathrm{a}$ disease following SARS-CoV-2 infection. SARS-CoV-2 viral protein-specific antibody levels were determined as described in the legend of Figure 1 in 23 individuals carrying the CCR5 delta 32 mutation heterozygously and in 105 individuals carrying the wild type CCR5 gene (Panel A). Disease duration in individuals with $\mathrm{WHO}^{\circ} 1$ and $\mathrm{WHO}^{\circ}$ 2a disease following SARS-CoV-2 infection is shown in Panel $\mathrm{B}$ for 8 heterozygous carriers of the CCR5 delta mutation and 39 non carriers.
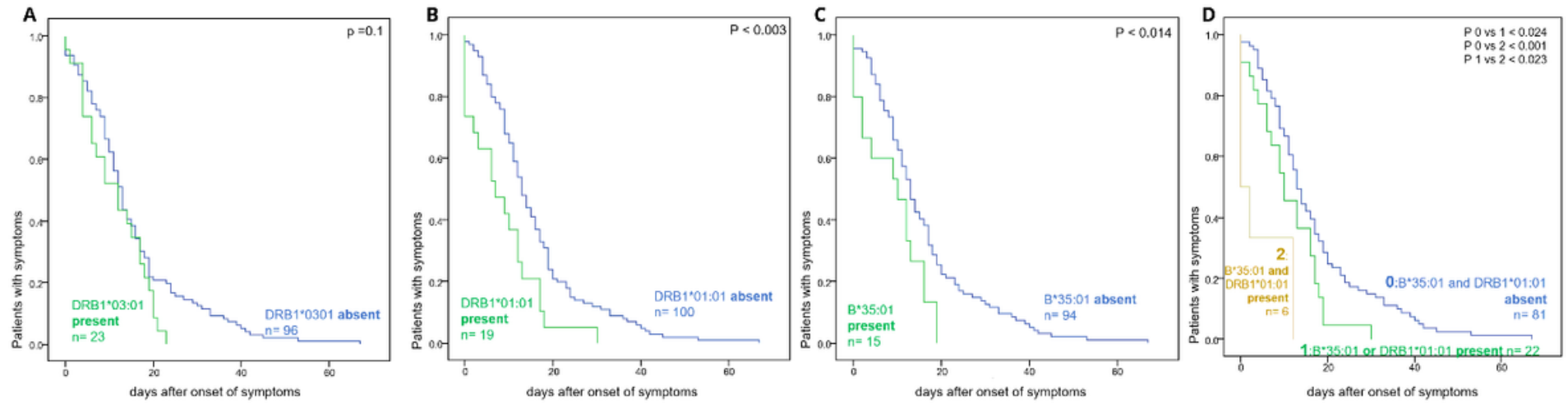

Figure 4

Association of HLA-DRB1*03:01, HLA-DRB1*01:01 and/ or HLA-B*35:01 allele expression with shorter COVID19 disease duration in SARS-CoV-2 infected individuals Panel A shows the disease duration related to HLA DRB1* 03:01 expression, Panel B shows the disease duration related to HLA*DBR1*01:01 expression, Panel $C$ shows disease duration related to HLA B* $35: 01$ expression. Panel D shows the disease duration related to the combination of HLA: DRB1*01:01 and or HLA B* 35:01 expression. 0 indicates the 81 individuals who express neither allele, (1) indicates the 22 individuals who express HLA: 
DRB1*01:01 $(n=13)$ or HLA B* 35:01 allele $(n=9)$, and (2) indicates the 6 individuals expressing both alleles.

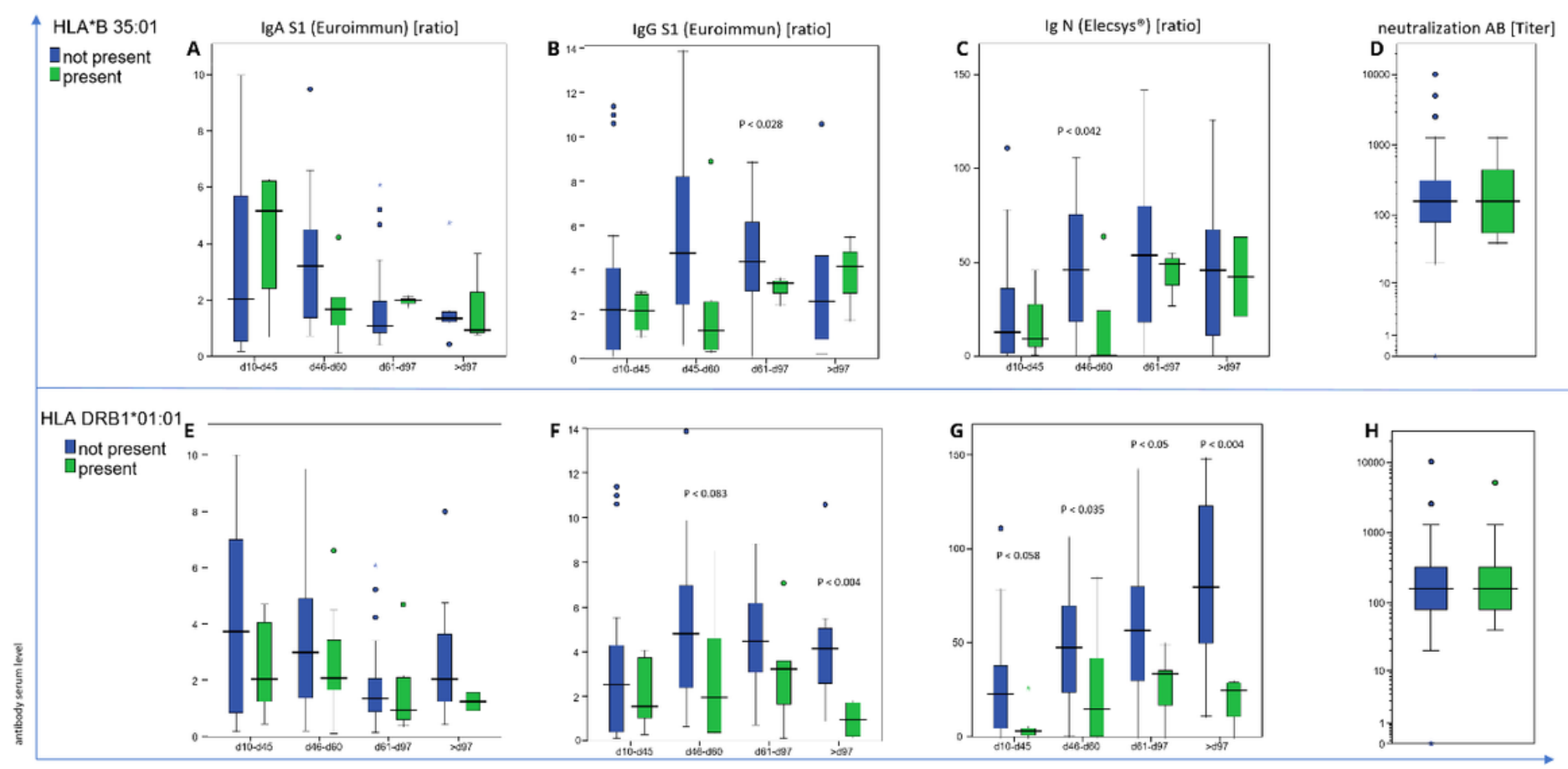

days after symptom onset

\section{Figure 5}

Association of "protective" HLA alleles with SARS-CoV-2 viral protein-specific antibody levels. Panel A and Panel E show similar levels of S1 specific IgA antibodies in the individuals who express "protective" HLA alleles and those who do not. Panel B and Panel C show a significantly lower level of S1 specific IgG antibodies and $\mathrm{N}$ specific antibodies in the 15 individuals who express the HLA-B $* 35: 01$ allele (green) than in the 94 individuals who do not express (blue) Panel F and Panel G show a significantly lower level of $\mathrm{S} 1$ specific IgG antibodies and $\mathrm{N}$ specific antibodies in the 19 individuals who express the HLADRB1*01:01 allele (green) than in the 100 individuals who do not express (blue) Panel D and Panel H show no detectable difference in the titer of neutralizing antibodies between individuals who express "protective" HLA alleles and those who do not. 

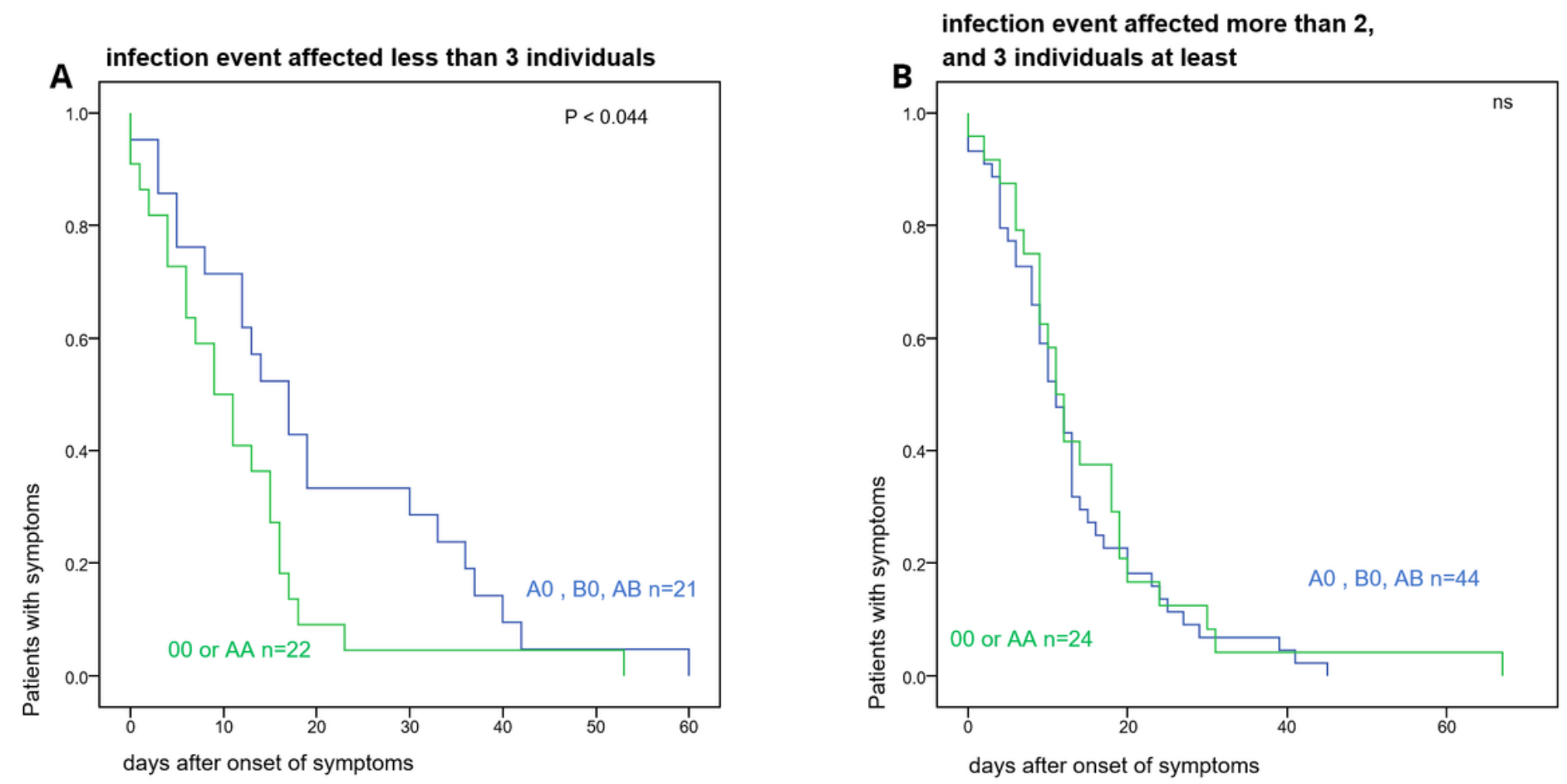

Figure 6

Effect of concomitant infection on the association of $\mathrm{AB} 0$ blood group heterozygosity with prolonged disease duration Panel $A$ shows the disease duration in 43 individuals in whom infection event affected less than 3 individuals. Panel B shows disease duration in 68 individuals in whom the infection event affected at least 3 individuals.

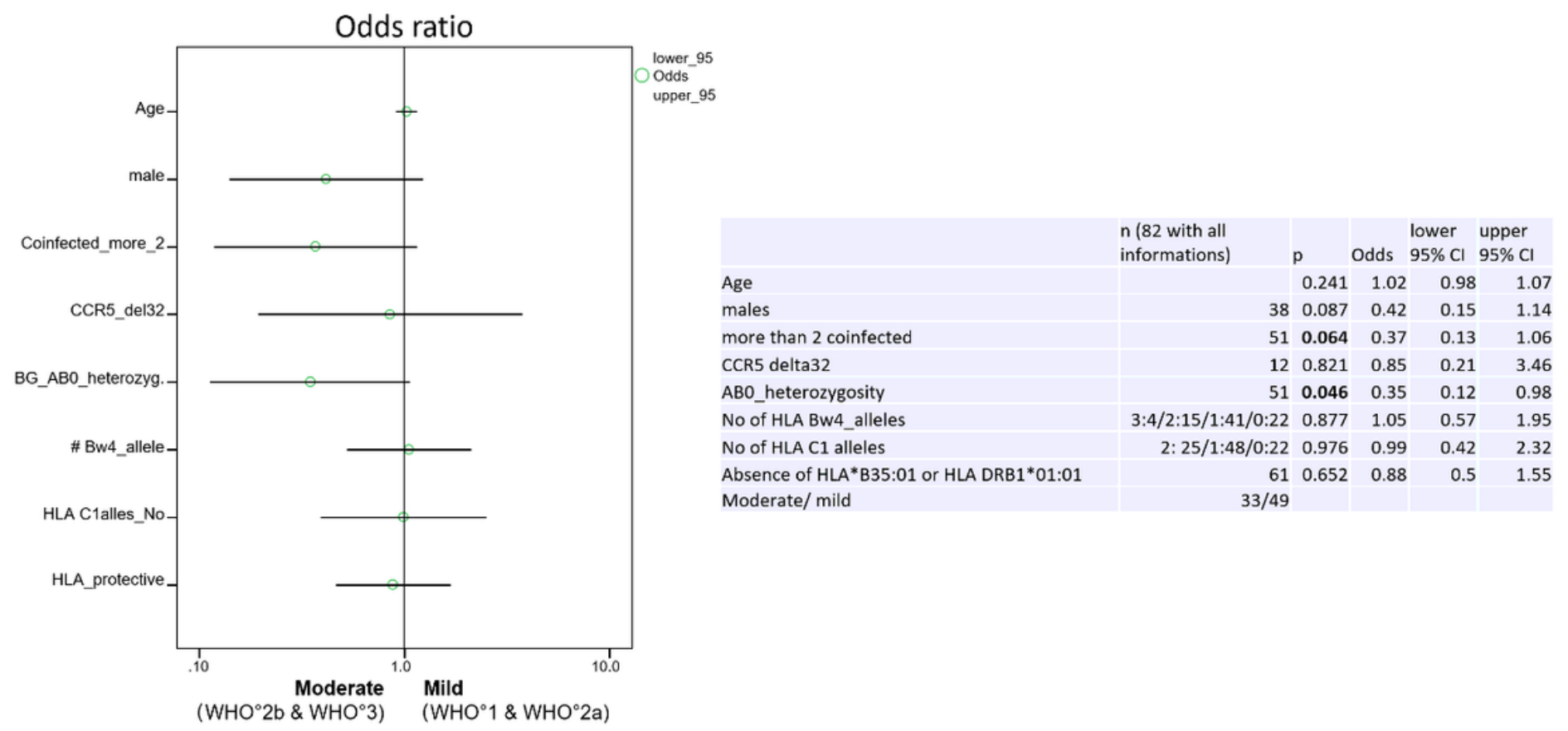

Figure 7 
Association of $\mathrm{ABO}$ blood group heterozygosity with development of $\mathrm{WHO}^{\circ} 2 \mathrm{~b}$ and $\mathrm{WHO} 3$ disease $\mathrm{A}$ logistic regression analysis included as covariates gender, age, multispreading (whether infection event affected at least 3 individuals), "protective" HLA alleles (as defined on the Results section), the further class I HLA alleles C2 and HLA Bw4 (oncluded on this analysis for their binding to distinct KIRs expressed by natural killer-cells), the heterozygous CCR5 delta 32 mutation and AB0 blood group allele heterozygosity. Odds ratios and their confidence intervals for individuals with COVID $19 \mathrm{WHO}^{\circ} 1$ to $\mathrm{WHO}^{\circ} 3$ are shown.
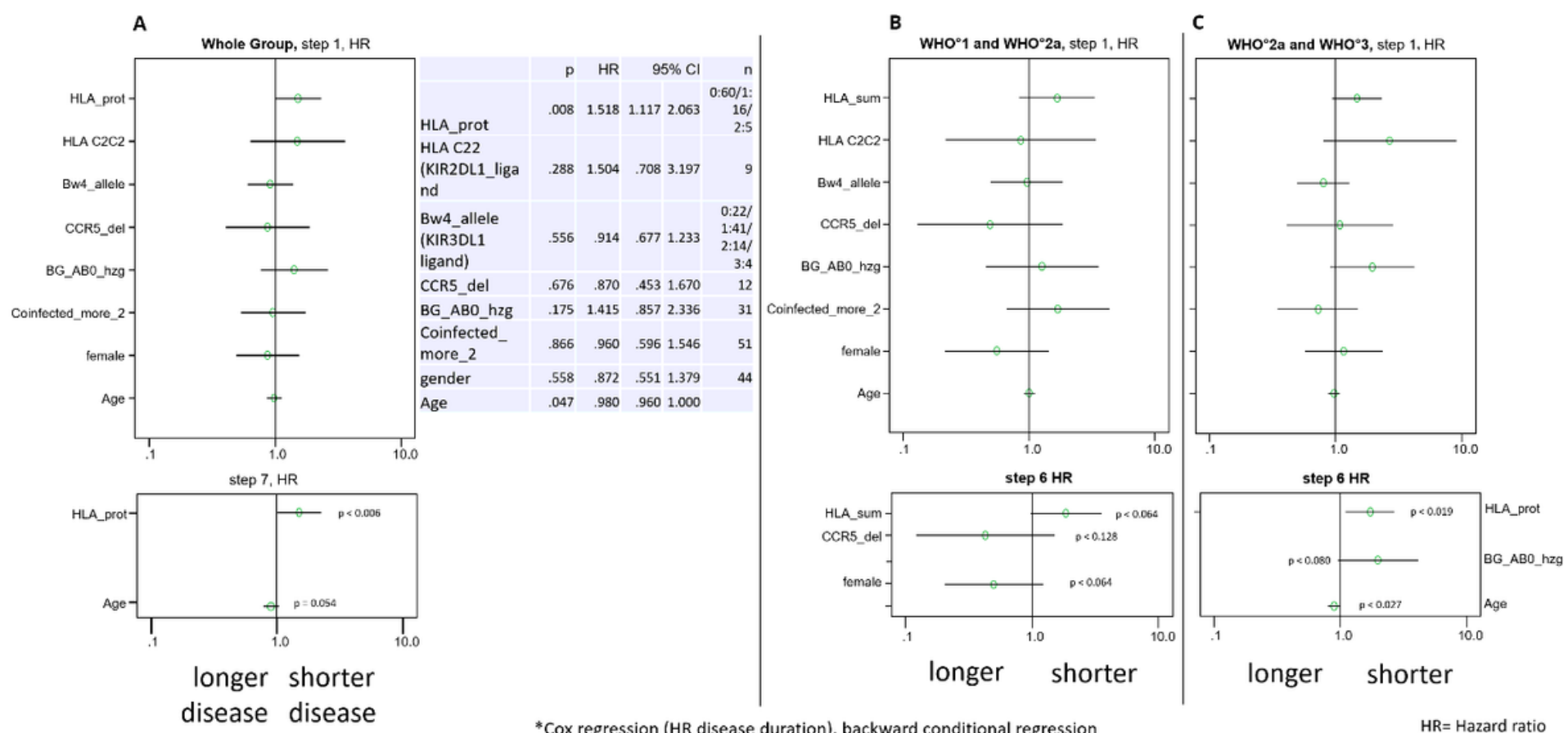

* Cox regression (HR disease duration). backward conditional regression

$H R=$ Hazard ratio

\section{Figure 8}

HLA alleles as most informative prognostic biomarkers of disease duration A Cox regression analysis included as covariates gender, age, number of "protective" HLA alleles, HLA encoded NK-KIR ligand for KIR2DL1/ KIR2DS1 (HLA C2) and KIR3DL1/KIR3DS1 (HLA Bw4), heterozygous CCR5 delta 32 mutation, as well as $\mathrm{ABO}$ blood group allele homozygosity. Panel $\mathrm{A}$ shows the results of a Cox regression analysis performed in 81 individuals for whom all the listed information is available. Panel $B$ shows the results of a Cox regression analysis performed in 51 individuals with $\mathrm{WHO}^{\circ} 1$ and $\mathrm{WHO}^{\circ} 2 \mathrm{a}$ disease. Panel $\mathrm{C}$ shows the results of a Cox regression analysis performed in 30 individuals with $\mathrm{WHO}^{\circ} 2 \mathrm{a}$ and $\mathrm{WHO}^{\circ} 3$ disease. Hazard ratio for disease duration and respective $95 \%$ confidence interval is shown for the first step and the last step after backward conditional regression.

\section{Supplementary Files}

This is a list of supplementary files associated with this preprint. Click to download.

- Onlinefloatimage2.png 
- Onlinefloatimage6.png 